\title{
EXEMPLARY DELINEATION OF OSMF TURNING MALICIOUS
}

\author{
Anjali Narwal ${ }^{1}$, Mala Kamboj ${ }^{2}$, Shruti Gupta ${ }^{3}$, Esha Singh ${ }^{4}$, Pooja Sharma ${ }^{5}$ \\ ${ }^{1}$ Associate Professor, Department of Oral \& Maxillofacial Pathology, Post Graduate Institute of Dental Sciences, Haryana, India \\ ${ }^{2}$ Senior Professor and Head, Department of Oral \& Maxillofacial Pathology, Post Graduate Intitute of Dental Sciences, Haryana, India \\ ${ }^{3}$ Assistant Professor, Department of Oral Anatomy, Post Graduate Institute of Dental Sciences, Haryana, India \\ ${ }^{4}$ Demonstrator, Department of Oral \& Maxillofacial Pathology, Post Graduate Institute of Dental Sciences, Haryana, India \\ ${ }^{5}$ Post Graduate Student, Department of Oral \& Maxillofacial Pathology, Post Graduate Institute of Dental Sciences, Haryana, India
}

Corresponding Author: Anjali Narwal

anjalinarwal@yahoo.com Received: $3^{\text {rd }}$ March 2017

Accepted: $20^{\text {th }}$ July 2017 Online: $20^{\text {th }}$ September 2017

\begin{abstract}
Oral submucous fibrosis (OSMF) is a well-recognized, potentially malignant condition of the oral cavity that can affect any part of the oral mucosa. It is strongly associated with a risk of oral cancer, although the biology underlying this association is still unresolved. The purpose of this paper is to show the histopathological prototypical illustration of OSMF turning into oral squamous cell carcinoma.
\end{abstract}

Keywords: Malignant transformation, Oral submucous fibrosis, Oral squamous cell carcinoma.

Oral submucous fibrosis (OSMF) is an insidious chronic disease affecting any part of the oral cavity and sometimes the pharynx. It is occasionally preceded by and or associated with vesicle formation, but always associated with juxtra-epithelial inflammatory reaction followed by a fibroelastic changes in the lamina propria, with epithelial atrophy leading to stiffness of the oral mucosa, causing trismus and inability to eat. ${ }^{1}$

Clinically, the presence of palpable fibrous bands is a diagnostic criterion for the disease. Once bands are palpable it means fibrosis has set in OSMF and, it will not regress spontaneously even on cessation of areca nut chewing. ${ }^{2}$ There are essentially two outcomes of established OSMF: persistence without significant progression of disease or eventual malignant transformation. OSMF is strongly associated with a risk of oral cancer, although the biology underlying this association is still unresolved. ${ }^{3}$ Studies suggest that dysplasia is seen in about $25 \%$ of biopsied OSMF cases and the rate of transformation to malignancy varies from $3 \%$ to $19 \%{ }^{4}$ About 2.5 million people are affected worldwide, with most cases being in southern India because of the areca nut chewing habit. ${ }^{5}$

The purpose of this paper is to show the prototypical illustration of OSMF turning into oral Squamous cell carcinoma (OSCC). Although numerous scientific papers and variable malignant transformation rate has been reported in literature but very few studies have shown this histopathological transformation in one picture.

In the indexed case, 37-year-old male patient presented with the complaint of restricted mouth opening and inability to chew as well as swallow properly since 2 years. His personal history revealed that 
he had been chewing tobacco (gutka packet) since 15 years with consumption of 6-8 packets per day. Patient went to a private practitioner few months back for the same problem where no surgical or pharmacological intervention was done. He was only advised to stop this habit immediately after which he had partially quit the same. Since no medication was given so he came as regular outdoor patient in Post Graduate Institute of Dental Sciences, Rohtak with the chief complaint of painful and restricted mouth opening. Complete history and clinical details were noted. Only vertical fibrous bands were palpable in the buccal mucosa bilaterally. Patient had dull aching pain while opening the mouth and it was restricted to $14-15 \mathrm{~mm}$. Intraorally partial blanching of mucosa with slight erythematous/ inflamed areas were evident (Figure 1). Haematological investigations showed haemoglobin content of $10 \mathrm{mg} / \mathrm{dl}$ with normal bleeding and clotting time.

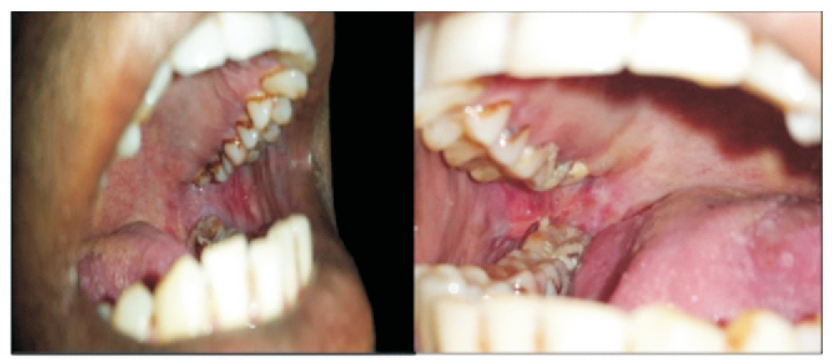

Figure 1: Intraoral picture depicting partial blanching of mucosa with slight erythematous/inflamed areas

Management of OSMF includes cessation of habit and surgical release of fibrous bands followed by forceful opening of the mouth by fibrotomy and coverage of surgical defects with nasolabial flap and postoperative active jaw physiotherapy for atleast 6 months. ${ }^{6}$ Although in scientific literature as well as in clinical practice there are two debatable school of thoughts i.e whether to biopsy OSMF or not. In the present case also clinically no ulceration or cancerous changes were observed but pain and mild erythema convinced the surgeon to take a small bit of tissue for histopathological confirmation. Simultaneously other medications and supplements were advised for palliative treatment of mouth opening.
Informed consent was taken from the patient. A small punch biopsy was conducted and tissue was sent for histopathological evaluation. On microscopic examination, tissue section revealed overlying dysplastic stratified squamous epithelium with variable dysplasia ranging from mild to moderate, severe and even carcinoma in situ at places. Underlying connective tissue was intensely collagenized with mild chronic inflammation. Subepithelial hyalinization with loss of rete pegs or stretched epithelium was evident. In few areas dysplastic epithelial islands had invaded the underlying stroma. Deeper connective tissue showed more compactly arranged collagen bundles (Figure 2).

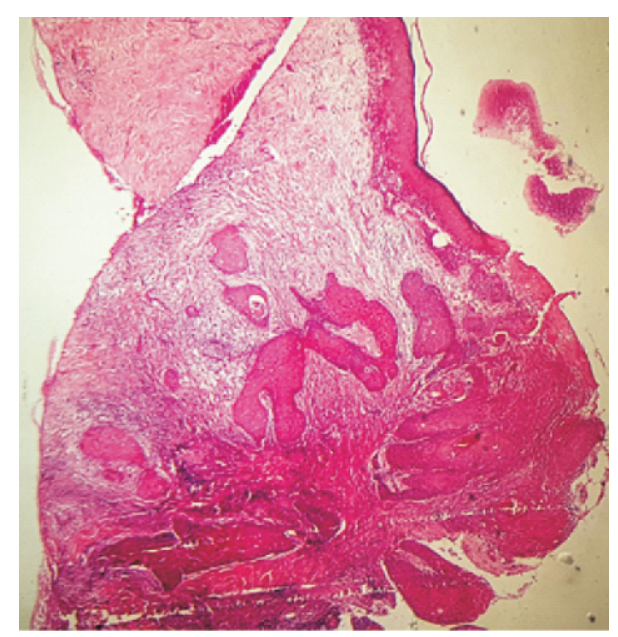

Figure 2: Histopathological picture depicting dysplastic stratified squamous epithelium with loss of rete pegs. At one focus, dysplastic epithelial islands had invaded the underlying stroma. Deeper connective tissue shows more compactly arranged collagen bundles.(H\&E 10x)

Histopathological picture was exemplary where in the same focus all features of OSMF as well as OSCC were seen. Diagnosis of OSMF converting into OSCC was made. The patient didn't return for further management after incisional biopsy.

Paymaster first described the malignant potential of OSMF in 1956 and since then various studies have been carried out to evaluate rate of transformation which currently ranges from $7-13 \%$.

Recently discovered mechanism involved in OSMF converting into squamous cell carcinoma as proposed by Ranganathan et al suggests that through 
the process of collagen maturation and its interaction with myofibroblasts and mast cells plays a role in this transformation. Submucosal fibrosis cause vascular constriction which results in epithelial hypoxia and has also been proposed as an important mechanism for the malignant transformation of OSMF. ${ }^{8}$

The habitual use of betel products from childhood, duration of use, frequency and cumulative amount all being factors, enhance the risk of early development of OSMF and, with progression to more lethal variants, increases the probability of transformation to frank OSCC.

\section{REFERENCES}

1. Pillai R, Balaram P. Pathogenesis of oral submucous fibrosis: relationship to risk factors associated with oral cancer. Cancer 1992;69:2011-20

2. Hammer JE, Mehta FS; eds. Tobacco related oral mucosal lesions and conditions in India. Mumbai: Basic Dental
Research Unit, Tata Institute of Fundamental Research; 1993.

3. Mithani SK, Mydlarz WK, Grumbine FL, Smith IM, Califano JA. Molecular genetics of premalignant oral lesions. Oral Dis 2007; 13: 126-33.

4. Murti PR, Bhonsle RB, Pindborg JJ, Daftary DK, Gupta PC, Metha FS. Malignant transformation rate in oral sub mucous fibrosis over a 17-year period. Community Dent Oral Epidemiol 1985; 13:340-1.

5. Gupta PC, Hebert JR, Bhonsle RB, Sinor PN, Mehta H, Mehta FS. Dietary factors in oral leukoplakia and submucous fibrosis in a population-based case control study in Gujarat, India. Oral Dis 1998;4:200-6.

6. Borle RM, Nimonkar PV. Extended nasolabial flaps in the management of oral submucous fibrosis. British Journal of Oral and Maxillofacial Surgery. 2009;47:382-5.

7. Paymaster JC. Cancer of buccal mucosa- clinical study of 650 cases in Indian Patients. Cancer 1956; 9: 431-5.

8. Ray JG, Ranganathan K, Chattopadhaya A. Malignant transformation of oral submucous fibrosis: overview of histopathological aspects. Oral Surg Oral Med Oral Pathol Oral Radiol 2016;122(2):200-9. 